\title{
Measuring interstellar magnetic fields by radio synchrotron emission
}

\author{
Rainer Beck \\ Max-Planck-Institut für Radioastronomie, Auf dem Hügel 69, 53121 Bonn, Germany \\ email: rbeck@mpifr-bonn.mpg.de
}

\begin{abstract}
Radio synchrotron emission, its polarization and its Faraday rotation are powerful tools to study the strength and structure of interstellar magnetic fields. The total intensity traces the strength and distribution of total magnetic fields. Total fields in gas-rich spiral arms and bars of nearby galaxies have strengths of $20-30 \mu$ Gauss, due to the amplification of turbulent fields, and are dynamically important. In the Milky Way, the total field strength is about $6 \mu \mathrm{G}$ near the Sun and several $100 \mu \mathrm{G}$ in filaments near the Galactic Center. - The polarized intensity measures ordered fields with a preferred orientation, which can be regular or anisotropic fields. Ordered fields with spiral structure exist in grand-design, barred, flocculent and even in irregular galaxies. The strongest ordered fields are found in interarm regions, sometimes forming "magnetic spiral arms" between the optical arms. Halo fields are X-shaped, probably due to outflows. - The Faraday rotation of the polarization vectors traces coherent regular fields which have a preferred direction. In some galaxies Faraday rotation reveals large-scale patterns which are signatures of dynamo fields. However, in most galaxies the field has a complicated structure and interacts with local gas flows. In the Milky Way, diffuse polarized radio emission and Faraday rotation of the polarized emission from pulsars and background sources show many small-scale and large-scale magnetic features, but the overall field structure in our Galaxy is still under debate.
\end{abstract}

Keywords. Techniques: polarimetric - ISM: magnetic fields - galaxies: magnetic fields - galaxies: spiral - radio continuum: galaxies

\section{Introduction}

Interstellar magnetic fields were discovered already in 1932 by Karl Guthe Jansky who first detected diffuse low-frequency radio emission from the Milky Way, but the explanation as synchrotron emission was given only in 1950 by Karl Otto Kiepenheuer. The sensitivity of radio observations has improved by several orders of magnitude in the past decades, and synchrotron emission was detected from the interstellar medium (ISM) in almost all star-forming galaxies, in galaxy halos and the intracluster medium, proving that a large fraction of the Universe is permeated by magnetic fields. However, in spite of our increasing knowledge on interstellar magnetic fields, many important questions are unanswered, especially their first occurrence in young galaxies, their amplification when galaxies evolved, and their effect on galaxy dynamics.

As magnetic fields need illumination by cosmic-ray electrons to become observable by synchrotron emission, which are generated in star-forming regions or intracluster shocks, we do not know yet whether magnetic fields also exist in radio-quiet elliptical or dwarf galaxies or in the general intergalactic medium (IGM). Progress can be expected from using Faraday rotation which does not need cosmic rays, only magnetic fields and thin ionized gas. One of the research areas of the forthcoming radio telescopes (LOFAR, ASKAP, SKA) will be the search for Faraday rotation in these objects against polarized background sources (Gaensler, this volume). The SKA will also be needed to detect magnetic fields in young galaxies (Beck \& Gaensler 2004, Arshakian et al. 2008). 


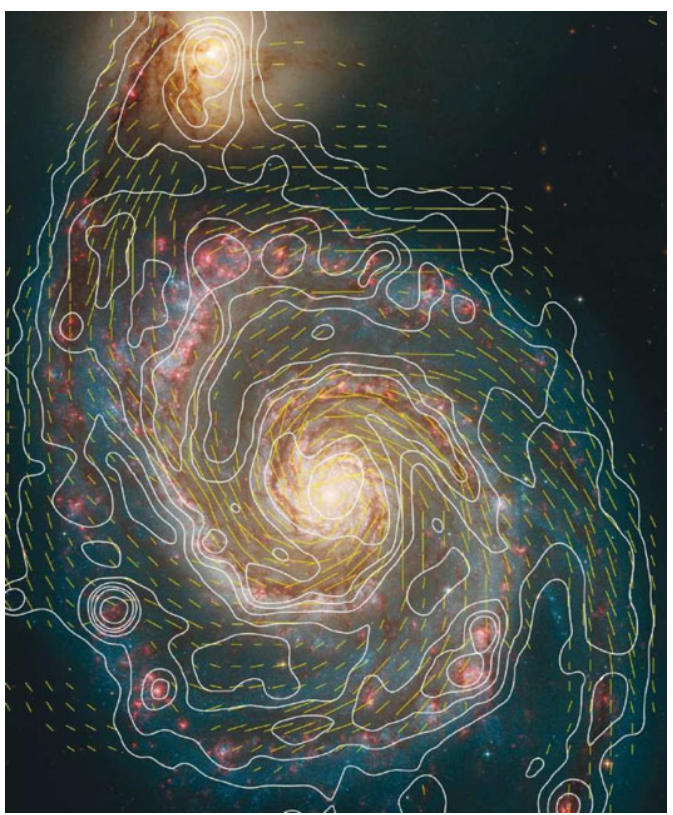

Figure 1. Total radio emission (contours) and $B$-vectors of M 51, combined from observations at $6 \mathrm{~cm}$ wavelength with the VLA and Effelsberg telescopes and smoothed to 15" resolution (Fletcher et al., in prep.), overlaid onto an optical image from the HST (Copyright: MPIfR Bonn and Hubble Heritage Team. Graphics: Sterne und Weltraum).

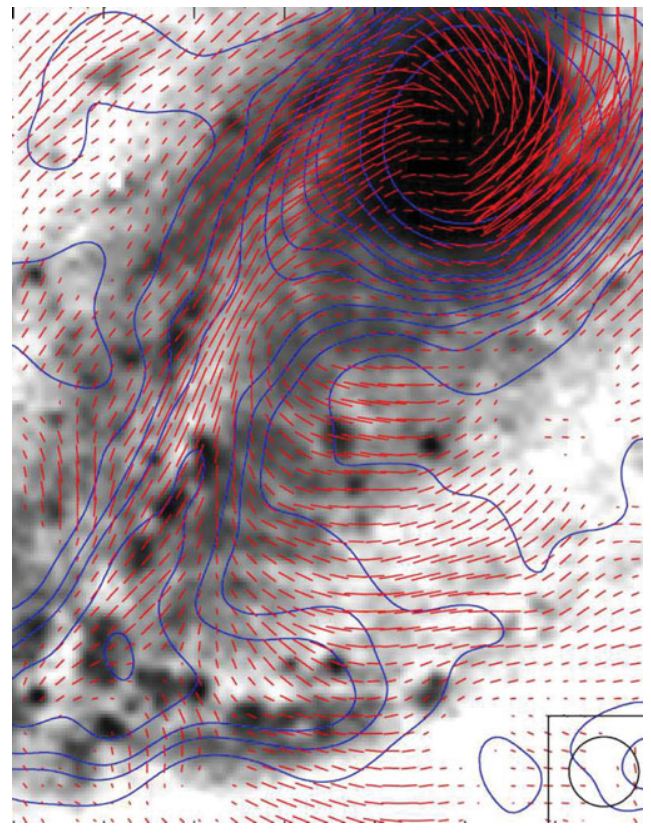

Figure 2. Total radio emission (contours) and $B$-vectors of the barred galaxy NGC 1097, observed at $6 \mathrm{~cm}$ wavelength with the VLA and smoothed to 10" resolution (Beck et al. 2005). The background optical image is from Halton Arp (Copyright: MPIfR Bonn and Cerro Tololo Observatory).

\section{Tools to measure interstellar magnetic fields}

Most of what we know about galactic and intergalactic magnetic fields comes through the detection of radio waves. Zeeman splitting of radio spectral lines is the best method to directly measure the field strength (Heiles, this volume).

The intensity of synchrotron emission is a measure of the number density of cosmicray electrons in the relevant energy range and of the strength of the total magnetic field component in the sky plane. Polarized emission emerges from ordered fields. As polarization "vectors" are ambiguous by $180^{\circ}$, they cannot distinguish regular fields with a constant direction within the telescope beam from anisotropic fields which are generated from turbulent magnetic fields by compressing or shearing gas flows and frequently reverse their direction on small scales. Unpolarized synchrotron emission indicates turbulent fields with random directions which have been tangled or generated by turbulent gas flows.

The intrinsic degree of linear polarization of synchrotron emission is about $75 \%$. The observed degree of polarization is smaller due to the contribution of unpolarized thermal emission, which may dominate in star-forming regions, by Faraday depolarization along the line of sight and across the beam (Sokoloff et al. 1998), and by depolarization due to variations of the field orientation within the beam and along the line of sight.

At short (e.g. centimeter) radio wavelengths the orientation of the observed $B$-vector is generally parallel to the field orientation, so that the magnetic patterns of many galaxies could be mapped directly (Beck 2005). The orientation of the polarization vectors is changed in a magnetized thermal plasma by Faraday rotation. The rotation angle 
increases with the plasma density, the strength of the component of the field along the line of sight and the square of the observation wavelength. As the rotation angle is sensitive to the sign of the field direction, only regular fields can give rise to Faraday rotation, while anisotropic and random fields do not. For typical plasma densities and regular field strengths in the interstellar medium of galaxies, Faraday rotation becomes significant at wavelengths larger than a few centimeters. Measurements of the Faraday rotation from multi-wavelength observations allow to determine the strength and direction of the regular field component along the line of sight. Its combination with the total intensity and the polarization vectors can yield the three-dimensional picture of the magnetic field and allows to distinguish the three field components: regular, anisotropic and random.

\section{Total synchrotron emission: Tracer of star formation}

The integrated flux densities of total radio continuum emission at centimeter wavelengths (frequencies of a few $\mathrm{GHz}$ ), which is mostly of non-thermal synchrotron origin, and far-infrared (FIR) emission of star-forming galaxies are tightly correlated, first reported by de Jong et al. (1985). This correlation achieved high popularity in galactic research as it allows to use radio continuum emission as a extinction-free tracer of star formation. The correlation holds for starburst galaxies (Lisenfeld et al. 1996b) as well as for blue compact and low-surface brightness galaxies (Chyży et al. 2007). It extends over five orders of magnitude (Bell 2003) and is valid to redshifts of at least 3 (Seymour et al. 2008), so that radio emission serves as a star formation tracer in the early Universe. Only galaxies with very recent starbursts reveal significantly smaller radio-to-FIR ratios because the timescale for the acceleration of cosmic rays and/or for the amplification of magnetic fields is longer than that of dust heating (Roussel et al. 2003).

Strongest total synchrotron emission (tracing the total, mostly turbulent field) generally coincides with highest emission from dust and gas in the spiral arms: The correlation also holds for the local radio and FIR or mid-IR (MIR) intensities within galaxies (e.g. Beck \& Golla 1988, Hoernes et al. 1998, Walsh et al. 2002, Tabatabaei et al. 2007a). The highest correlation of all spectral ranges is found between the total intensity at $\lambda 6 \mathrm{~cm}$ and the mid-infrared dust emission, while the correlation with the cold gas (traced by the $\mathrm{CO}(1-0)$ transition) is less tight (Frick et al. 2001, Walsh et al. 2002, Nieten et al. 2006). A wavelet cross-correlation analysis for M 33 showed that the radio-FIR correlation holds for all scales down to $1 \mathrm{kpc}$ (Tabatabaei et al. 2007a). The correlation breaks down below scales of about $50 \mathrm{pc}$ (Hughes et al. 2006) and in radio halos, probably due to the smoothing effect on synchrotron intensity by cosmic-ray propagation.

If the thermal and non-thermal radio components are separated with help of $\mathrm{H} \alpha$ and FIR data (e.g. within M 33, Tabatabaei et al. 2007b), an almost perfect correlation is found between thermal radio and infrared intensities at all scales. The nonthermalFIR correlation is less pronounced but highly significant. The polarized synchrotron intensity, tracing the ordered field, is anticorrelated or not correlated with all tracers of star formation (Frick et al. 2001).

It is not obvious why the nonthermal synchrotron and the thermal FIR intensities are so closely related. The intensity of synchrotron emission depends not only on the density of cosmic-ray electrons (CRE), but also on about the square of the strength of the total magnetic field $B_{\mathrm{t}}$ (its component in the sky plane, to be precise). The radio-FIR correlation requires that magnetic fields and star-formation processes are connected. If $B_{\mathrm{t}}$ is strong, most of the cosmic-ray energy is released via synchrotron emission within the galaxy, the CRE density decreases with $B_{\mathrm{t}}^{2}$ and the integrated radio synchrotron 
luminosity depends on the CRE injection rate, not on $B_{\mathrm{t}}$. If most thermal energy from star formation is also emitted within a galaxy via far-infrared emission by warm dust, this galaxy can be treated as a "calorimeter" for thermal and nonthermal emission. Prime candidates for "calorimeter" galaxies are those with a high star-formation rate (SFR). If $B_{\mathrm{t}}$ increases with SFR according to $B_{\mathrm{t}} \propto S F R^{0.5}$, a linear radio-FIR correlation for the integrated luminosities is obtained (Lisenfeld et al. 1996a,b). However, the calorimeter model cannot explain the local correlation within galaxies.

In galaxies with low or moderate SFR and $B_{\mathrm{t}}$, synchrotron lifetime of CRE is sufficiently large to leave the galaxy. To obtain a global or local radio-FIR correlation, coupling of magnetic fields to the gas clouds is needed. A scaling $B_{\mathrm{t}} \propto \rho^{1 / 2}$ was proposed (Helou \& Bicay 1993, Niklas \& Beck 1997, Hoernes et al. 1998) where $\rho$ is the average density of the neutral gas. A nonlinear correlation (with a slope of about 1.3) between the nonthermal radio luminosity and the FIR luminosity from warm dust is achieved by further assuming energy equipartition between magnetic fields and cosmic rays and a Schmidt law of star formation $\left(S F R \propto \rho^{1.5}\right)$ (Niklas \& Beck 1997). In this model the total magnetic field strength and the star-formation rate $S F R$ are related via $B_{\mathrm{t}} \propto S F R^{0.3}$.

The radio-FIR correlation indicates that equipartition between the energy densities of the total magnetic field and the total cosmic rays is valid, at least on spatial scales larger than about $100 \mathrm{pc}$ (Stepanov et al., this volume) and on timescales of larger than the CRE acceleration time (a few $10^{6}$ years). Then the strength of the total magnetic field can be determined from the intensity of the total synchrotron emission, assuming a ratio $K$ between the numbers of cosmic-ray protons and electrons in the relevant energy range (usually $K \simeq 100$ ). In regions where electrons lost already a significant fraction of their energy, e.g. in strong magnetic fields or radiation fields or far away from their places of origin, $K$ is $>100$ and the standard value of 100 yields an underestimate (Beck \& Krause 2005). On the other hand, in case of field fluctuations along the line of sight or across the telescope beam, the equipartition value is an overestimate (Beck et al. 2003).

The typical average equipartition strength of the total magnetic field in spiral galaxies is about $10 \mu \mathrm{G}$. Radio-faint galaxies like M 31 and M 33, our Milky Way's neighbors, have weaker total magnetic fields (about $5 \mu \mathrm{G}$ ), while gas-rich galaxies with high starformation rates, like M 51 (Fig. 1), M 83 and NGC 6946, have total field strengths of 20$30 \mu \mathrm{G}$ in their spiral arms. The degree of radio polarization within the spiral arms is only a few \%; hence the field in the spiral arms must be mostly tangled or randomly oriented within the telescope beam, which typically corresponds to a few $100 \mathrm{pc}$. Turbulent fields in spiral arms are probably generated by turbulent gas motions related to supernovae (de Avillez \& Breitschwerdt 2005), stellar winds, spiral shocks (Dobbs \& Price 2008) or a small-scale turbulent dynamo (Beck et al. 1996).

The mean energy densities of the magnetic field and of the cosmic rays in NGC 6946 and M 33 are $\simeq 10^{-11} \mathrm{erg} \mathrm{cm}^{-3}$ and $\simeq 10^{-12} \mathrm{erg} \mathrm{cm}^{-3}$, respectively (Beck 2007, Tabatabaei et al. 2008 and this volume), about 10 times larger than that of the ionized gas, but similar to that of the turbulent gas motions across the whole star-forming disk. The magnetic energy possibly dominates in the outer disk of NGC 6946.

The strongest total fields of $50-100 \mu \mathrm{G}$ are found in starburst galaxies, like M 82 (Klein et al. 1988) and the "Antennae" NGC 4038/9 (Chyży \& Beck 2004), and in nuclear starburst regions, like in the centers of NGC 1097 and other barred galaxies (Beck et al. 2005). In starburst galaxies, however, the equipartition field strength per average gas surface density is much lower than in normal spirals. This indicates strong energy losses of the cosmic-ray electrons, so that the equipartition field strength is probably underestimated by a factor of a few (Thompson et al. 2006). This was recently confirmed by Zeeman measurements of $\mathrm{OH}$ maser lines (Robishaw et al. 2008). 


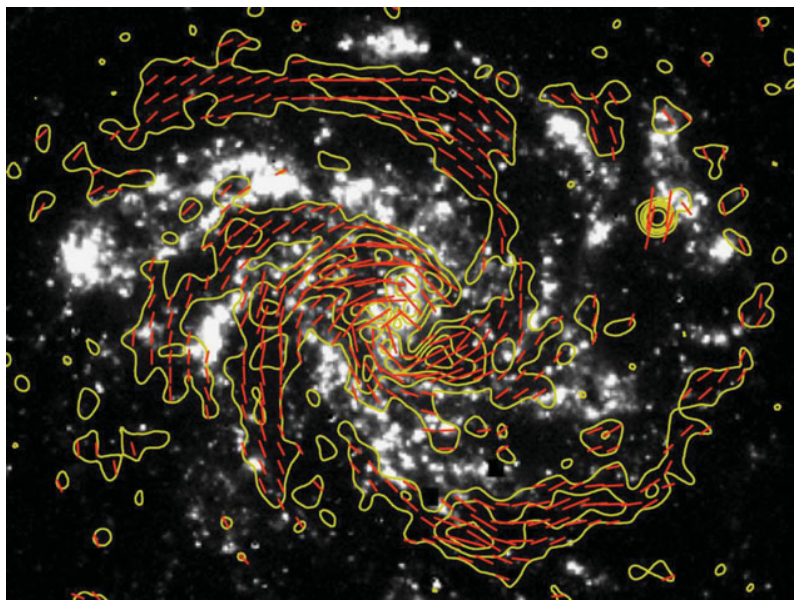

Figure 3. Polarized radio emission (contours) and $B$-vectors of NGC 6946 (15" resolution), combined from observations at $6 \mathrm{~cm}$ wavelength with the VLA and Effelsberg $100 \mathrm{~m}$ telescopes (Beck \& Hoernes 1996). The background image shows the $\mathrm{H} \alpha$ emission (Ferguson et al. 1998) (Copyright: MPIfR Bonn. Graphics: Sterne und Weltraum).

In case of energy equipartition, the scale length of the total field in the disk of galaxies is at least $(3-\alpha)$ times larger than the synchrotron scale length of typically $4 \mathrm{kpc}$ (where $\alpha \simeq-1$ is the synchrotron spectral index). The resulting value of $\simeq 16 \mathrm{kpc}$ is a lower limit because the CRE lose their energy with distance from the star-forming disk and the equipartition assumption yields too small values for the field strength. The galactic fields extend far out into intergalactic space. The same argument holds for the vertical extent of radio halos around galaxies which is also limited by CRE energy losses. The dumbbell-shaped halo around edge-on galaxies like NGC 253 (Heesen et al. 2008 and this volume) is the result of enhanced synchrotron and Inverse Compton losses in the inner region.

As proposed by Battaner \& Florido (2000), the magnetic field energy density may generally reach the level of global rotational gas motion and affect galaxy rotation in the outermost parts of spiral galaxies. At $\mathrm{GHz}$ frequencies the measured extent of the radio disks of galaxies is limited by energy loss of cosmic-ray electrons, so that measurements at low frequencies (where energy losses are smaller) are needed, e.g. with LOFAR (Beck 2008). Faraday rotation towards polarized background sources may allow to measure weak fields to even larger distances from the star-forming disks.

\section{Polarized synchrotron emission: Tracer of ordered fields}

The ordered (regular and/or anisotropic) fields traced by the polarized synchrotron emission are generally strongest $(10-15 \mu \mathrm{G})$ in the regions between the optical spiral arms, oriented parallel to the adjacent optical spiral arms. In some galaxies the field forms magnetic arms between the optical arms, like in NGC 6946 (Fig. 3). These are probably generated by a large-scale dynamo. In galaxies with strong density waves some of the ordered field is concentrated at the inner edge of the spiral arms, e.g. in M 51 (Patrikeev et al. 2006), but the arm-interarm contrast of the ordered field is small, much smaller than that of the random field.

The ordered magnetic field forms spiral patterns in almost every galaxy (Beck 2005), even in ring galaxies (Chyży \& Buta 2008) and in flocculent galaxies without an optical spiral structure (Soida et al. 2002). Hence, the field lines generally do not follow the (almost circular) gas flow and need dynamo action to obtain the required radial field components. Spiral fields with large pitch angles are also observed in the central regions of galaxies and in circum-nuclear gas rings (Beck et al. 2005). 


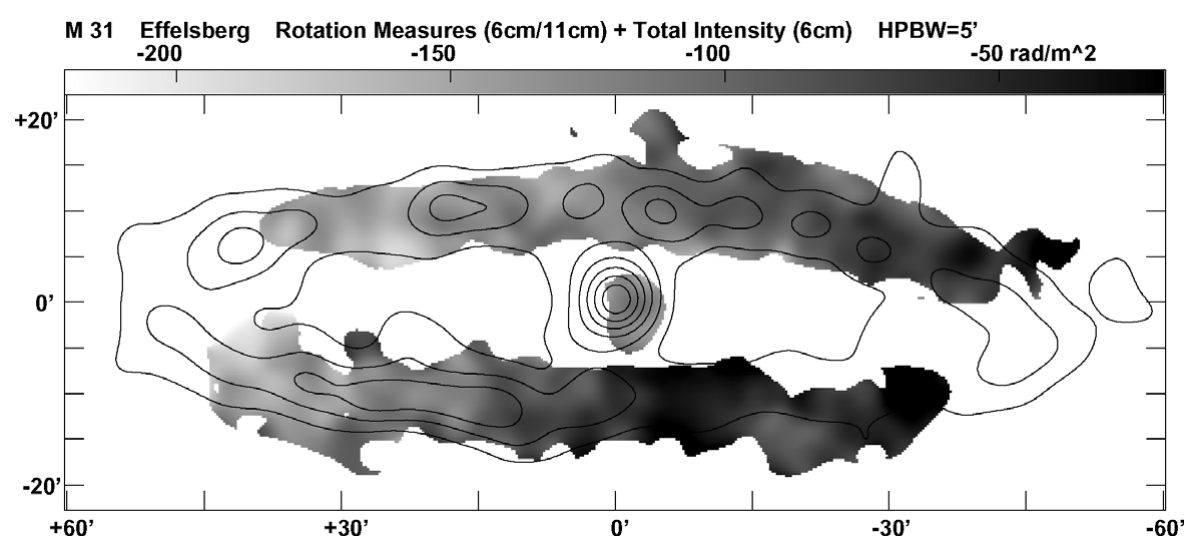

Figure 4. Total radio emission (contours) at $6 \mathrm{~cm}$ and Faraday rotation measures (RM) between $6 \mathrm{~cm}$ and $11 \mathrm{~cm}$ of the Andromeda galaxy M 31 (5' resolution), observed with the Effelsberg telescope (Berkhuijsen et al. 2003) (Copyright: MPIfR Bonn).

In galaxies with massive bars the field lines follow the gas flow (Fig. 2). As the gas rotates faster than the bar pattern of a galaxy, a shock occurs in the cold gas which has a small sound speed, while the flow of warm, diffuse gas is only slightly compressed but sheared. The ordered field is also hardly compressed. It is probably coupled to the diffuse gas and strong enough to affect its flow (Beck et al. 2005, Fletcher et al., this volume). The polarization pattern in spiral arms and bars can be used as a tracer of shearing gas flows in the sky plane and hence complements spectroscopic measurements.

Nearby galaxies seen edge-on generally show a disk-parallel field near the disk plane (Dumke et al. 1995), so that polarized emission can also be detected from distant, unresolved galaxies (Stil et al. 2008). High-sensitivity observations of edge-on galaxies like NGC 891 (Krause 2008) and NGC 253 (Heesen et al., this volume) revealed vertical field components in the halo forming an X-shaped pattern (Krause, this volume). This is inconsistent with the predictions from standard dynamo models. The field is probably transported from the disk into the halo by an outflow emerging from the disk. The similarity of scale heights of radio halos in different galaxies indicates that the outflow velocity increases with the star-formation rate (Krause, this volume). Interestingly, a recent model for global outflows from galaxy disks (neglecting magnetic fields) shows an X-shaped velocity field (Dalla Vecchia \& Schaye 2008). Improved outflow models including magnetic fields and dynamo action are needed.

\section{Faraday rotation: Tracer of regular dynamo fields}

Faraday rotation is a signature of regular (coherent) fields which could be generated by the mean-field (or large-scale) dynamo (Elstner et al. 1992, Beck et al. 1996; Elstner, this volume). Dynamo fields are described by modes of different azimuthal symmetry in the disk plane and vertical symmetry (even or odd parity) perpendicular to the disk plane. Several modes can be excited in the same object. In flat, rotating objects like galaxy disks, the strongest mode S0 consists of a toroidal field of axisymmetric spiral shape within the disk, without sign reversals across the equatorial plane, and a weaker poloidal field of quadrupolar structure with a reversal of the vertical field component across the plane. Antisymmetric (A-type) fields are generated preferably in spherical objects like halos (Moss \& Sokoloff 2008). The magneto-rotational instability (MRI) supports symmetric fields while primordial seed fields support antisymmetric fields (Krause \& Beck 1998). 
Spiral dynamo modes can be identified from the pattern of polarization angles and Faraday rotation measures (RM) from multi-wavelength radio observations of galaxy disks (Krause 1990, Elstner et al. 1992) or from RM data of polarized background sources (Stepanov et al. 2008). The disks of a few spiral galaxies indeed reveal large-scale RM patterns, as predicted. The Andromeda galaxy M 31 hosts a dominating axisymmetric disk field (mode S0) (Fig. 4 and Fletcher et al. 2004). Other candidates for a dominating axisymmetric disk field are the nearby spiral IC 342 (Krause et al. 1989) and the irregular Large Magellanic Cloud (LMC) (Gaensler et al. 2005). The magnetic arms in NGC 6946 can be described by a superposition of two azimuthal dynamo modes which are phase shifted with respect to the optical arms (Beck 2007). However, in many observed galaxy disks no clear patterns of Faraday rotation were found. Either several dynamo modes are superimposed and cannot be distinguished with the limited sensitivity and resolution of present-day telescopes, or the timescale for the generation of large-scale modes is longer than the galaxy's lifetime. Dynamo models predict a rapid amplification of smallscale fields already in protogalaxies, while the generation of fully coherent large-scale fields takes several Gyrs, depending on the galaxy size (Arshakian et al. 2008 and this volume). Furthermore, anisotropic fields dominate over dynamo modes if shearing gas flows are strong (see Sect. 4).

Vertical fields as predicted by dynamo models should generate large-scale RM patterns around edge-on galaxies, but so far indication for an antisymmetric poloidal field was found only in the halo of NGC 253 (Heesen et al., this volume). Previous indirect evidence for symmetric fields from the dominance of inward-directed fields (Krause \& Beck 1998) is no longer supported by the increased sample of galaxies.

Faraday rotation in the direction of QSOs allows to determine the field pattern in an intervening galaxy (Stepanov et al. 2008). This method can be applied to much larger distances than the analysis of RM of the polarized emission from the foreground galaxy itself. Faraday rotation of QSO emission in distant, intervening galaxies revealed significant regular fields of several $\mu \mathrm{G}$ strength (Bernet et al. 2008, Kronberg et al. 2008) which is a challenge for classical dynamo models (Arshakian et al. 2008).

The recently developed method of RM Synthesis, based on multi-channel spectropolarimetry, transforms the spectral data cube into a data cube of maps in Faraday depth space (Brentjens \& de Bruyn 2005, Heald, this volume). RM components from distinct regions along the line of sight can be distinguished by their positions and widths in Faraday depth. Faraday screens appear as sharp lines, while emitting and rotating layers form broad structures in Faraday depth. The transformation of Faraday depth into geometrical depth (Faraday tomography) needs modeling.

\section{Magnetic fields in the Milky Way}

Surveys of the total synchrotron emission from the Milky Way yield equipartition strengths of the total field of $6 \mu \mathrm{G}$ near the Sun and about $10 \mu \mathrm{G}$ in the inner Galaxy (Berkhuijsen, in Wielebinski 2005), consistent with Zeeman splitting data of low-density gas clouds (Heiles, this volume). In the nonthermal filaments near the Galactic center the field strength may reach several $100 \mu \mathrm{G}$ (Reich 1994). Milligauss fields were found in pulsar wind nebulae from the break in the synchrotron spectrum (e.g., Kothes et al. 2008).

The observed degree of radio and optical polarization in the local Galaxy implies a ratio of ordered to total field strengths of $\simeq 0.6$ (Heiles 1996). For a total field of $6 \mu \mathrm{G}$ this gives $4 \mu \mathrm{G}$ for the local ordered field component (including anisotropic fields). Faraday $\mathrm{RM}$ and dispersion measure data of pulsars give an average strength of the local coherent 


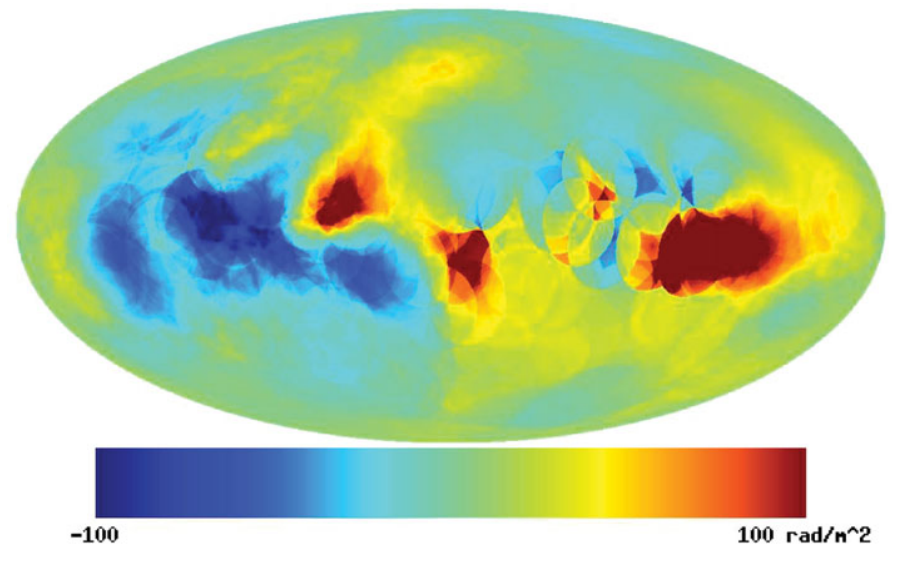

Figure 5. All-sky map of rotation measures in the Milky Way, generated from data towards about 800 polarized extragalactic sources compiled by Johnston-Hollitt et al. (2004) and from about 1800 data of the new Effelsberg RM survey (from Sun et al. 2008).

regular field of $1.4 \pm 0.2 \mu \mathrm{G}$ (Rand \& Lyne 1994). In the inner Norma arm, the average strength of the coherent regular field is $4.4 \pm 0.9 \mu \mathrm{G}$ (Han et al. 2002).

The all-sky maps of polarized synchrotron emission at $1.4 \mathrm{GHz}$ from the Milky Way from DRAO and Villa Elisa and at $22.8 \mathrm{GHz}$ from WMAP, and the new Effelsberg RM survey of polarized extragalactic sources were used to model the large-scale Galactic field (Sun et al. 2008). One large-scale reversal is required about 1-2 kpc inside the solar radius, which also agrees with the detailed study of RMs from extragalactic sources near the Galactic plane (Brown et al. 2007, Kothes \& Brown, this volume). Recent pulsar RM values indicate reversals at several Galactic radii (Han, this volume). None of the models for a simple large-scale field structure of the Milky Way survived a statistical test (Men et al. 2008). Similar to external galaxies, the Milky Way's regular field probably has a complex structure which can be revealed only based on a larger sample of pulsar and extragalactic RM data.

RMs of extragalactic sources and of pulsars reveal no large-scale reversal across the plane for Galactic longitudes $1=90^{\circ}-270^{\circ}$ (Fig. 5): the local disk field is part of a largescale symmetric (quadrupolar) field structure. However, towards the inner Galaxy $\left(\mathrm{l}=270^{\circ}-\right.$ $90^{\circ}$ ) the RM signs are opposite above and below the plane (Fig. 5). This may indicate a global antisymmetric mode in the halo (Han et al. 1997), similar to the results for the spiral galaxy NGC 253 (Heesen et al., this volume). Note that a superposition of a disk field with even parity and a halo field with odd parity cannot be explained by classical dynamo theory (Moss \& Sokoloff 2008).

While the large-scale field is much more difficult to measure in the Milky Way than in external galaxies, Galactic observations can trace magnetic structures to much smaller scales (Wielebinski 2005, Reich \& Reich, this volume). Small-scale and turbulent field structures are enhanced in spiral arms compared to interarm regions, as indicated by the different slopes of their RM structure functions (Haverkorn et al. 2006). The all-sky and the Galactic plane polarization surveys at $1.4 \mathrm{GHz}$ (Reich \& Reich and Landecker et al., this volume) reveal a wealth of structures on pc and sub-pc scales: filaments, canals, lenses and rings. Their common property is to appear only in the maps of polarized intensity, but not in total intensity. Some of these are artifacts due to strong depolarization of background emission in a foreground Faraday screen, called Faraday ghosts, but carry valuable information about the turbulent ISM (Fletcher \& Shukurov 2006). Faraday rotation in foreground objects (e.g. supernova remnants, planetary nebulae, pulsar wind nebulae or photo-dissociation regions of molecular clouds) embedded in diffuse polarized Galactic emission may generate a Faraday shadow or Faraday screen which enable to 
estimate the regular field strength by modeling the radiation transfer (Wolleben \& Reich 2004, Ransom et al. 2008, Reich \& Reich, this volume).

\section{Outlook}

Future radio telescopes will widen the range of observable magnetic phenomena. Highresolution, future deep observations at high frequencies with the Extended Very Large Array (EVLA) and the Square Kilometre Array (SKA) (Gaensler, this volume) will directly show the detailed field structure and the interaction with the gas. The SKA will also measure the Zeeman effect in much weaker magnetic fields in the Milky Way and nearby galaxies. Forthcoming low-frequency radio telescopes like the Low Frequency Array (LOFAR) and the Murchison Widefield Array (MWA) will be suitable instruments to search for extended synchrotron radiation at the lowest possible levels in outer galaxy disks, halos and clusters, and the transition to intergalactic space. At low frequencies we will get access to the so far unexplored domain of weak magnetic fields in galaxy halos (Beck 2008). The detection of radio emission from the intergalactic medium will allow to probe the existence of magnetic fields in such rarified regions, measure their intensity, and investigate their origin and their relation to the structure formation in the early Universe. Low frequencies are also ideal to search for small Faraday rotation from weak interstellar and intergalactic fields. "Cosmic magnetism" is the title of Key Science Projects for LOFAR and SKA.

\section{References}

Arshakian, T. G., Beck, R., Krause, M., \& Sokoloff, D. 2008, A\&A, in press, arXiv:0810.3114 Battaner, E. \& Florido, E. 2000, Fund. Cosmic Phys. 21, 1

Beck, R. 2005, in: R. Wielebinski, \& R. Beck (eds.), Cosmic Magnetic Fields, Lecture Notes in Physics (Berlin: Springer) vol. 664, p. 41

Beck, R. 2007, A\&A 470, 539

Beck, R. 2008, Rev. Mexicana AyA, in press, arXiv:0804.4594

Beck, R. \& Gaensler, B. M. 2004, New Astron. Revs 48, 1289

Beck, R. \& Golla, G. 1988, A\&A A 191, L9

Beck, R. \& Hoernes, P. 1996, Nature 379, 47

Beck, R. \& Krause, M. 2005, AN 326, 414

Beck, R., Brandenburg, A., Moss, D., Shukurov, A., \& Sokoloff, D. 1996, ARA\&A 34, 155

Beck, R., Shukurov, A., Sokoloff, D., \& Wielebinski, R. 2003, A\& A 411, 99

Beck, R., Fletcher, A., Shukurov, A., et al. 2005, A\&A 444, 739

Bell, E. F. 2003, ApJ 586, 794

Berkhuijsen, E. M., Beck, R., \& Hoernes, P. 2003, A\&A 398, 937

Bernet, M. L., Miniati, F., Lilly, S. J., Kronberg, P. P., \& Dessauges-Zavadsky, M. 2008, Nature 454,302

Brentjens, M. A. \& de Bruyn, A. G. 2005, A\& A 441, 1217

Brown, J. C., Haverkorn, M., Gaensler, B. M., et al. 2007, ApJ 663, 258

Chyży, K. T. \& Beck, R. 2004, A\&A 417, 541

Chyży, K. T. \& Buta, R. J. 2008, ApJ 677, L17

Chyży, K. T., Bomans, D. J., Krause, M., et al. 2007, A $\& A$ 462, 933

Dalla Vecchia, C. \& Schaye, J. 2008, MNRAS 387, 1431

de Avillez, M. A. \& Breitschwerdt, D. 2005, A\&A 436, 585

de Jong, T., Klein, U., Wielebinski, R., \& Wunderlich, E. 1985, A\&A 147, L6

Dobbs, C. L. \& Price, D. J. 2008, MNRAS 383, 497

Dumke, M., Krause, M., Wielebinski, R. \& Klein, U. 1995, A\&A 302, 691

Elstner, D., Meinel, R., \& Beck, R. 1992, A\& AS 94, 587 
Ferguson, A. M. N., Wyse, R. F. G., Gallagher, J. S., \& Hunter, D. A. 1998, ApJ 506, L19

Fletcher, A. \& Shukurov, A. 2006, MNRAS 371, L21

Fletcher, A., Berkhuijsen, E. M., Beck, R., \& Shukurov, A. 2004, A $\mathscr{E} A$ 414, 53

Frick, P., Beck, R., Berkhuijsen, E. M., \& Patrikeev, I. 2001, MNRAS 327, 1145

Gaensler, B. M., Haverkorn, M., Staveley-Smith, L., et al. 2005, Science 307, 1610

Han, J. L., Manchester, R. N., Berkhuijsen, E. M., \& Beck, R. 1997, A\&A A 322, 98

Han, J.L., Manchester, R. N., Lyne, A. G., \& Qiao, G. J. 2002, ApJ 570, L17

Haverkorn, M., Gaensler, B. M., Brown, J. C., et al. 2006, ApJ 637, L33

Heesen, V., Beck, R., Krause, M., \& Dettmar, R.-J. 2008, A\&A, in press, arXiv:0812.0346

Heiles, C. 1996, in: W. G. Roberge, \& D. C. B. Whittet (eds.), Polarimetry of the Interstellar Medium, ASP Conf. Ser. (San Francisco: Astron. Soc. Pac.), vol. 97, p. 457

Helou, G. \& Bicay, M. D. 1993, ApJ 415, 93

Hoernes, P., Berkhuijsen, E. M., \& Xu, C. 1998, A\& $A$ 334, 57

Hughes, A., Wong, T., Ekers, R., et al. 2006, MNRAS 370, 363

Johnston-Hollitt, M., Hollitt, C. P., \& Ekers, R.D. 2004, in: B. Uyaniker, W. Reich \& R. Wielebinski (eds.), The Magnetized Interstellar Medium (Katlenburg: Copernicus), p. 13

Kiepenheuer, K. O. 1950, Phys. Rev. 79, 738

Klein, U., Wielebinski, R. \& Morsi, H. W. 1988, A\& A 190, 41

Kothes, R., Landecker, T. L., Reich, W., Safi-Harb, S., \& Arzoumanian, Z. 2008, ApJ 687, 516

Krause, F. \& Beck, R. 1998, A\&SA 335, 789

Krause, M. 1990, in: R. Beck, R. Wielebinski, \& P. P. Kronberg (eds.), Galactic and Intergalactic Magnetic Fields (Dordrecht: Kluwer), p. 187

Krause, M. 2008, Rev. Mexicana AyA, in press, arXiv:0806.2060

Krause, M., Hummel, E., \& Beck, R. 1989, A\&A 217, 4

Kronberg, P. P., Bernet, M. L., Miniati, F., et al. 2008, ApJ 676, 70

Lisenfeld, U., Völk, H. J., \& Xu, C. 1996a, A $\& A$ 306, 677

Lisenfeld, U., Völk, H. J., \& Xu, C. 1996b, A\&A 314, 745

Men, H., Ferrière, K., \& Han, J. L. 2008, A\&SA 486, 819

Moss, D. \& Sokoloff, D. 2008, A\&A 487, 197

Nieten, C., Neininger, N., Guélin, M., et al. 2006, A\&A 453, 459

Niklas, S. \& Beck, R. 1997, A\&̋A 320, 54

Patrikeev, I., Fletcher, A., Stepanov, R., et al. 2006, A\&SA 458, 441

Rand, R. J. \& Lyne, A. G. 1994, MNRAS 268, 497

Ransom, R. R., Uyaniker, B., Kothes, R., \& Landecker, T. L. 2008, ApJ 684, 1009

Reich, W. 1994, in: R. Genzel, \& A. I. Harris (eds.), The Nuclei of Normal Galaxies (Dordrecht: Kluwer), p. 55

Robishaw, T., Quataert, E. \& Heiles, C. 2008, ApJ 680, 981

Roussel, H., Helou, G., Beck, R., et al. 2003, ApJ 593, 733

Seymour, N., Dwelly, T., Moss, D., et al. 2008, MNRAS 386, 1695

Soida, M., Beck, R., Urbanik, M. \& Braine, J. 2002, A\&A 394, 47

Sokoloff, D. D., Bykov, A. A., Shukurov, A., Berkhuijsen, E. M., Beck, R., \& Poezd, A. D. 1998, MNRAS 299, 189, and Erratum in MNRAS 303, 207

Stepanov, R., Arshakian, T. G., Beck, R., Frick, P., \& Krause, M. 2008, A\& A 480, 45

Stil, J. M., Krause, M., Beck, R., \& Taylor, A. R. 2008, ApJ, in press, arXiv:0810.2303

Sun, X. H., Reich, W., Waelkens, A., \& Enßlin, T. A. 2008, A\& A 477, 573

Tabatabaei, F., Beck, R., Krause, M., et al. 2007a, A\&A 466, 509

Tabatabaei, F., Beck, R., Krügel, E., et al. 2007b, A\&A 475, 133

Tabatabaei, F., Krause, M., Fletcher, A., \& Beck, R. 2008, A\&A 490, 1005

Thompson, T. A., Quataert, E., Waxman, E., Murray, N., \& Martin, C.L. 2006, ApJ 645, 186

Walsh, W., Beck, R., Thuma, G., et al. 2002, A\&SA 388, 7

Wielebinski, R. 2005, in: R. Wielebinski, \& R. Beck (eds.), Cosmic Magnetic Fields, Lecture Notes in Physics (Berlin: Springer) vol. 664, p. 89

Wolleben, M. \& Reich, W. 2004, A\&广A 427, 537 


\section{Discussion}

MAEDER: To what extend can the magnetic field you are observing contribute to the dynamics of the external regions of galaxies? In particular, is the field contribution to the flat rotation curve negligible or not?

BECK: Magnetic fields in external regions will certainly affect outflows and tide gas flows, as evidenced from existing data. However, the field strengths seem to be insufficient to affect outer rotation curves. Low-frequency observations with e.g. LOFAR should allow to measure magnetic fields to much larger radii.

HeILES: How good is the equipartition assumption? In particular, the derived field strength depends on the cosmic ray proton/electron ratio. How serious is this?

BECK: The equipartition field strength depends on the cosmic-ray proton/electron ration $K$ as about $K^{1 / 4}$, so that variations from the standard value of $K=100$ by a factor of several are not serious. However, in case of strong energy losses of electrons, $K$ may become very large, and the equipartition estimate underestimates the field strength. We need independent data on cosmic-ray protons and electrons, e.g. from $\gamma$-ray data.

HEILES: Why are synchrotron-derived field strengths so much larger than RM-derived fields? How about assumptions about equipartition?

BECK: Equipartition field strengths derived from total synchrotron intensity include turbulent fields, anisotropic (sheared) fields and regular (coherent) fields, whereas Faraday rotation traces regular fields only. In case of significant field fluctuations $\left(\delta B^{2} \geqslant B^{2}\right)$, the equipartition field strength is an overestimate. In case of fluctuations in regular field and ionized gas density, the Faraday-derived field strength is an underestimate if $\delta\left|B_{\text {reg }}\right|$ and $\delta n_{\mathrm{e}}$ are anticorrelated (see Beck et al. 2003 for details).

RüDIGER: Rainer, would you add, please, some comments about the equatorial parity of the ordered magnetic field? Is the quadrupolar parity now better established than in the past?

BECK: There is still no clear indications for quadrupolar or dipolar field patterns in galaxies because Faraday rotation measures in the halos of edge-on galaxies do not show large-scale patterns (but errors are large). We need better RM data, e.g. from LOFAR. In the Milky Way the local field is vertically symmetric (quadrupole), but the halo field is possibly antisymmetric (see Sun et al. 2008).

DE Gouveia dal Pino: Could you comment on the present status of the observations towards the potential bi-symmetric pattern in the Milky Way?

BECK: Please see the talks by A. Noutsos, J.L. Han and W. Reich for the present status of the interpretation of the existing data. In external galaxies a bisymmetric field was found only in M81 some time ago (Krause et al. 1989), but this is presently re-investigated with new data by M. Krause \& A. Fletcher. 


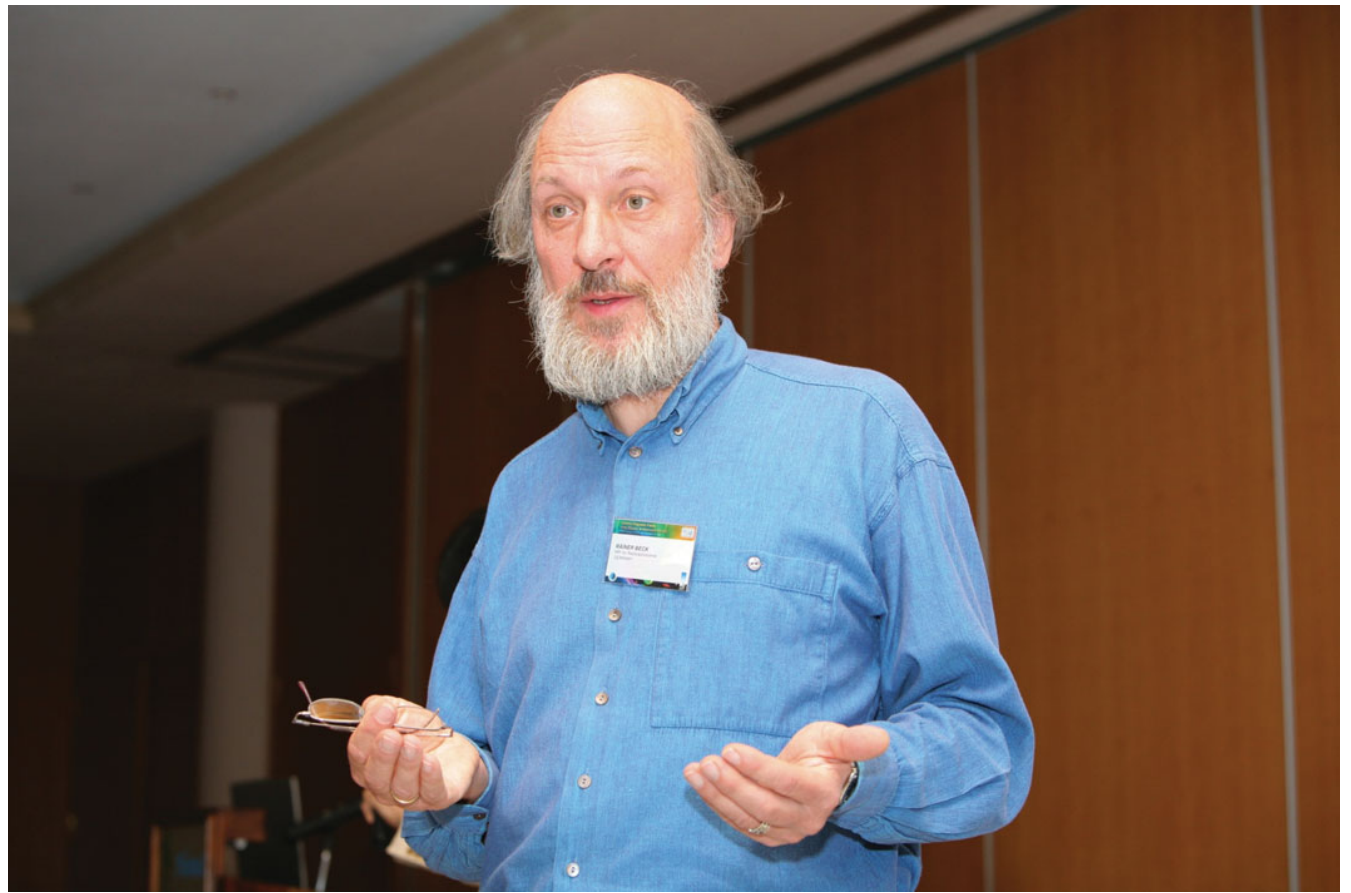

Rainer Beck

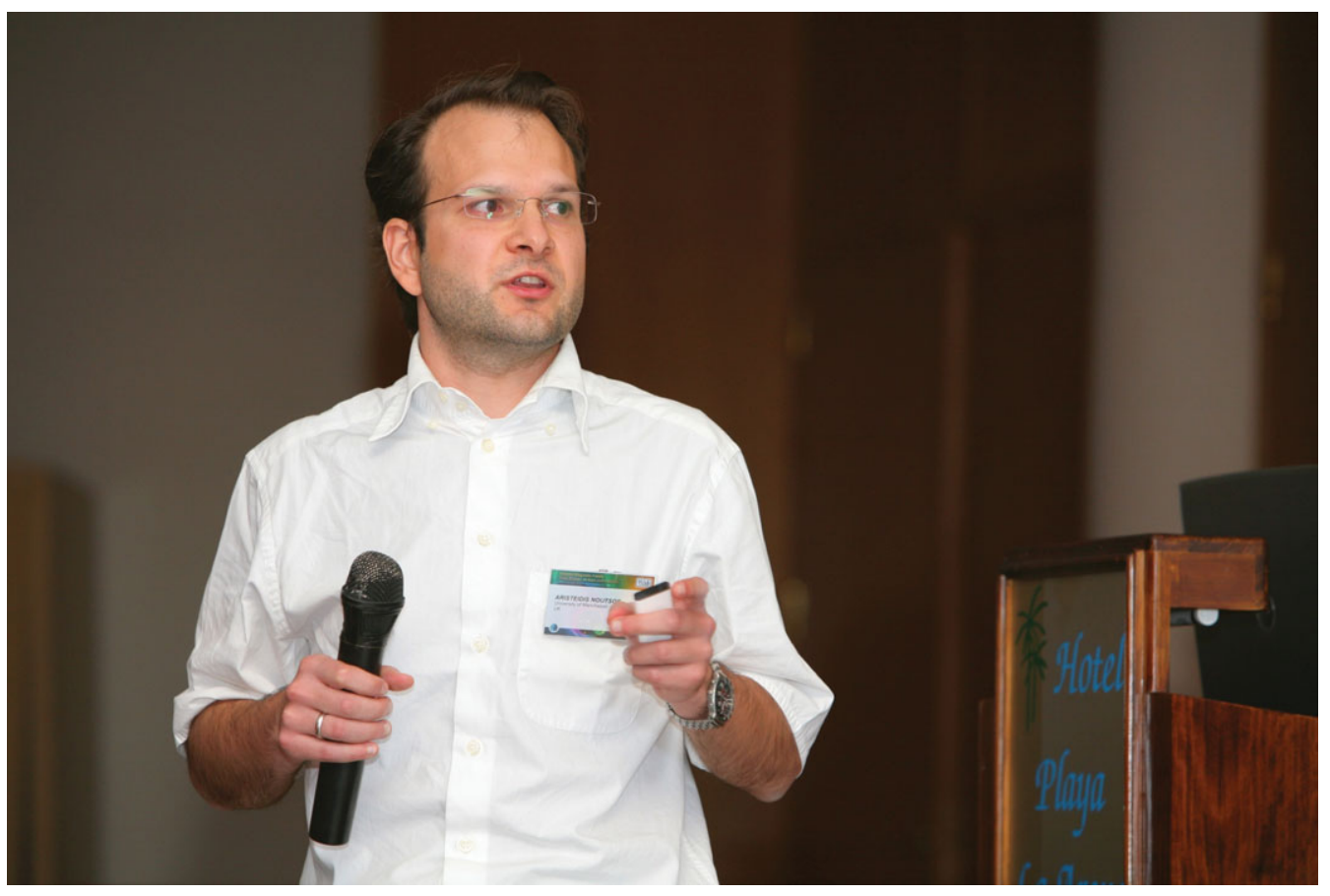

Aristeidis Noutsos 